
Military Technical College Kobry El-Kobbah, Cairo, Egypt.

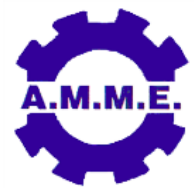
$17^{\text {th }}$ International Conference on Applied Mechanics and Mechanical Engineering.

\title{
SMALL LAUNCHER ENABLED BY HYBRID ROCKET MOTOR TECHNOLOGY
}

\author{
F. Mingireanu*
}

\begin{abstract}
In this paper, we present a small launcher for payloads $<10 \mathrm{~kg}$ and orbits of $350-400 \mathrm{~km}$ as well as for suborbital flights with larger payload. Currently there is an increasing demand for small launchers, demand that is increased especially by the nano-satellite expanding missions as well as a strategy shift to smaller and more efficient payloads. Advancements in electronics helped this direction by creating smaller and more powerful computers and communication equipments that can be fitted on smaller satellites with performances at least equal with the ones from the previous generation. We propose a three stage small launcher powered by solid-hybrid-hybrid combination. We investigate the full flight dynamics of the launcher with internal ballistic models for the hybrid rocket motors. The relatively new proposal is to use hybrid rocket motor technology for the second and third stage due to its lower cost of manufacturing as well as simplicity compared to liquid rocket motors. At the same time the performance promise from hybrid rocket motors and their throttable capabilities makes them ideal candidates for upper stage propulsion. Detailed discussion is performed related to the performance of hybrid rocket motors and its influence on launcher lift capability. We also show the preliminary design of such hybrid rocket motors as upper stage propulsion units and propose several fuel/oxidizer pairs based on both technical performances as well as cost parameters. LOX and solid methane are proposed as best oxidizer and fuel candidates in order to obtain a high specific impulse for the upper stages. Next we present a full 6 DOF model for the flight of the launcher as well as simulation results for various flight scenarios ending with different payloads on different orbits. Discussion is performed on guidance and control systems illustrating the main limitations and the option to use as many as possible COTS due to their availability and low design-production cycle. The paper ends with a discussion related to optimization procedures for a small launcher due to its importance on launcher performances and price of operation.
\end{abstract}

\section{KEYWORDS}

Rocket, hybrid, launcher, methane, flight, dynamics, altitude

* Research Scientist, Romanian Space Agency (ROSA), Bucharest, Romania E-mail: florin.mingireanu@rosa.ro. 


\section{NOMENCLATURE}

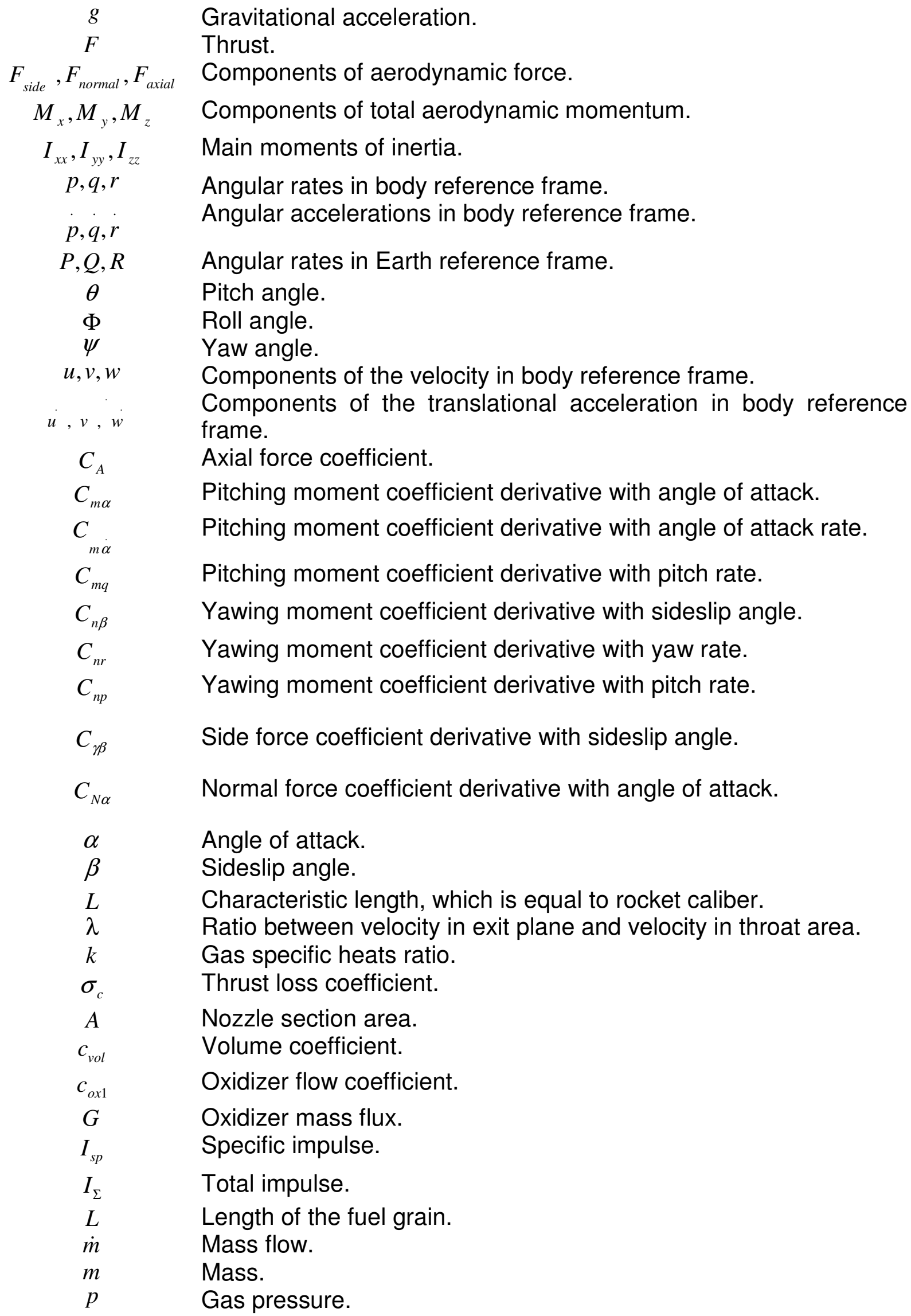




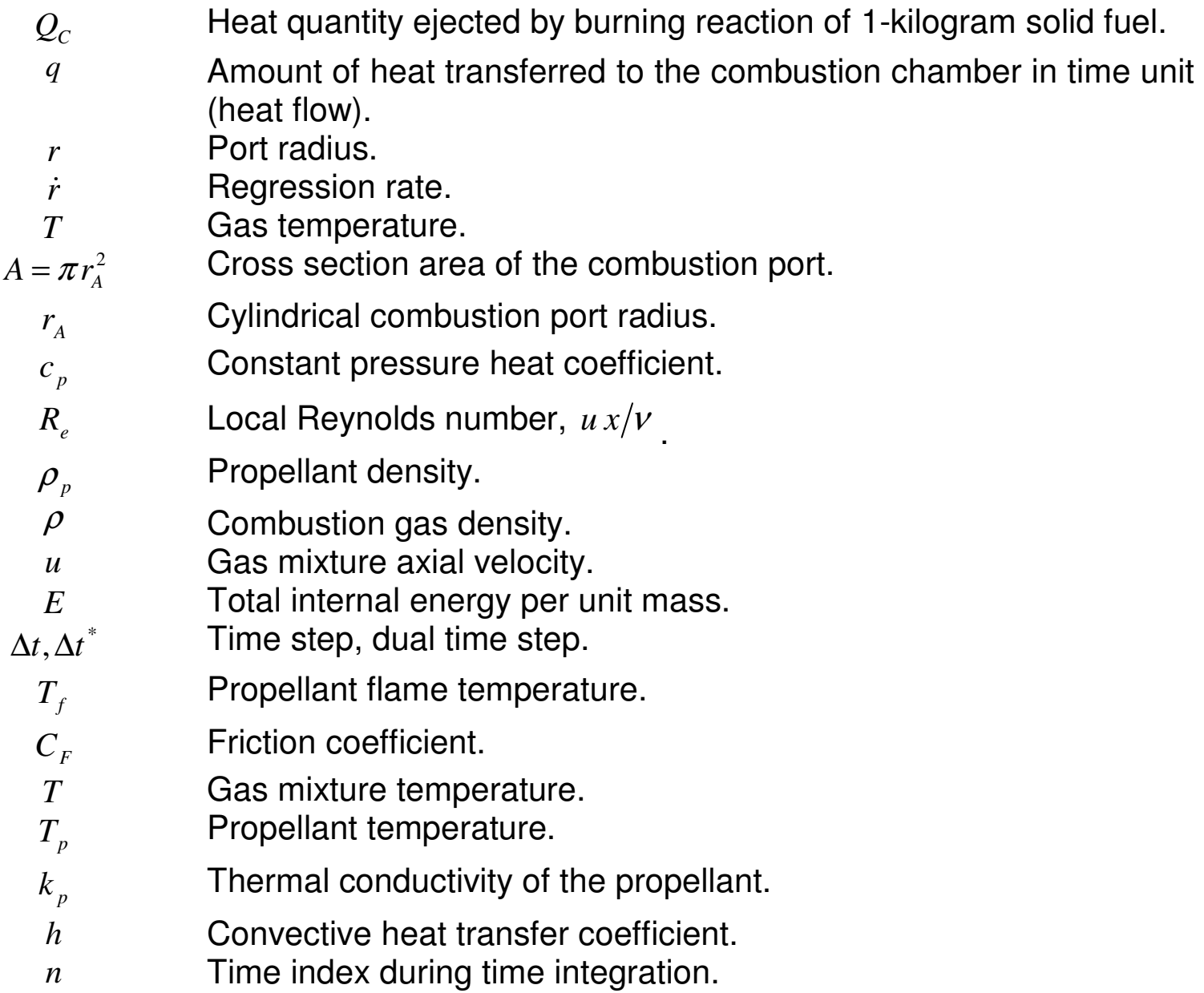

- All quantities are expressed using S.I. units.

\section{INTRODUCTION}

Satellite launchers are the workhorse of the entire space industry. Without reliable launchers, none of today's achievements in space could have been possible.

Initially most of the launchers were military ICBM converted for civilian usage. They were not designed for space application but rather were a good compromise for the time being and the large numbers of ICBMs offered a relatively cheap and fast access to space. One famous example of converted military ICBM is DNEPR which has been successfully used for LEO missions. However, the DNEPR stock is being used and there is a point in the future where no DNEPR will be available anymore.

Another category of launchers were ones designed and built specifically for space applications. These ones were characterized by quite large deployment costs because the design and tests were not covered by the military. They were part of the final cost of the launcher that had to be retrieved entirely from the operation of these launchers. A famous example is Ariane with continuous operation since the 1979 starting with Ariane 1 and ending with Ariane 6. They achieved a multitude of LEO and beyond the LEO missions with a good rate of success. GEO missions for telecommunication satellites are an important market segment for Ariane launcher 
vehicle family. However, all of these vehicles were designed for large satellites and spacecrafts (about tonnes or even larger).

In the last 10 years there has been a growing need of launching capabilities for small satellites: from cubesats to mini-satellites with masses smaller than $10 \mathrm{~kg}$. These satellites are becoming more and more capable for completing more complex missions at a much lower cost than their larger counterparts. It is well understood that the small satellite technology is still at the beginning but with miniaturization and optimization of humankind resources there will be a growing pressure to improve the capabilities of these small spacecrafts in order to fulfil tasks that were only approached by multi-tonne spacecrafts 20 years ago.

No current launchers are optimized for placing on LEO or beyond spacecrafts with masses of up to $10 \mathrm{~kg}$. There is the possibility to piggyback such a spacecraft on a larger launcher but in this case, the piggyback will be either restrained to the orbit of the mother mission or will have to carry its own propulsion unit large enough to modify its orbit from the initial mother orbit to the desired orbit. It is easy to see that in order to fly a small satellite on the desired orbit and to successfully complete its mission there are a lot of "if/then/else" iterations that which a mission designer has to take into account. A dedicated $<10 \mathrm{~kg}$ payload launcher would allow the mission designer to chooseexactly the orbit and the launching characteristics that are most beneficial for the small spacecraft.

Having the above considerations in mind we focused on proposing a launcher based on innovative hybrid rocket technology that is intended specifically for payloads of less than $10 \mathrm{~kg}$ and for LEO missions. Obviously, missions beyond LEO could be accommodated with modifications in the upper stages of the launcher: thrust level, burn-time and guidance and control flight program.

We approach in the following sections the internal ballistic model of HRM with emphasize on cryogenic solid methane HRM, the guidance through thrusters and the 6 DOF flight dynamics model applicable to the study of the launcher's trajectory.

The entire paper is just a proposal for a new launcher category that would fulfil a gap in the category of low mass spacecrafts. It does not intend to be a design manual for such a launcher but rather a starting point for future studies focused on each subcomponent of such a launcher.

\section{LAUNCHER STRUCTURE DESIGN}

The launcher envisioned is composed of three stages. First stage would be a solid rocket booster while the second and the third stage will have hybrid rocket motors on each of them. We choose the hybrid rocket motors due to their simplicity compared liquid rocket motors but the throttle ability compared with the solids.

However, due to high performance needed for the upper stages (in order to minimize the start mass of the vehicle) we choose solid methane hybrid rocket motors on both of the upper stages. These ensures specific impulses in excess of 310 seconds 
which places both propulsion units in the high performance area ensuring that each $\mathrm{kg}$ of start mass is well used to place the payload on the final orbit.

The entire structure is proposed to use lightweight aluminium alloys already used in aerospace applications both for storing the cryogenic fuels/oxidizers as well as for other components. The typical thrust and burn-times for each of the stages is shown in Table I.

As one can see for a total payload of $10 \mathrm{~kg}$ placed on a $400 \mathrm{~km}$ orbit the total initial launcher mass is of around $1200 \mathrm{~kg}$. This is a rather conservative figure since a typical launcher with similar specific impulse performance usually can place on LEO between $1 \%$ and $1.5 \%$ of its initial mass. Even higher percentages can be obtained but for initial studies it is better to choose the conservative figure.

The entire guidance of the launcher would be done through a combination of jet vanes and micro-thrusters.

Besides reaching the LEO it is obvious that such a launcher could also fulfil suborbital mission with larger payloads increasing the market share that it can fill-in and hence the revenues that it can generate.

\section{SOLID METHANE HRM}

\section{Internal Ballistic Model}

An important parameter in an internal ballistic model for a rocket motor is the burning rate of propellant. In the case of a hybrid rocket motor, the burning rate is called regression rate and is given by a relation indicated in previous works [1], [2], [3]

$$
\dot{r}=a G^{n} p^{m}(2 r)^{l}
$$

where $G$ is the oxidizer mass flux, $r$ is the radius of port combustion, $p$ is the pressure in the burning chamber, $a, n \quad m$ and $l$ are fuel coefficients determined by experimental burn tests for various pairs oxidizer/fuel.

In our model, we assume a cylindrical geometry for burning surfaces. Taking into account that:

$$
G=\frac{\dot{m}_{o x}}{\pi r^{2}}
$$

The regression rate relation (1) becomes:

$$
\dot{r}=a 2^{l} \pi^{-n} \dot{m}_{o x}^{n} p^{m} r^{l-2 n} .
$$

where $\dot{m}_{0 x}$ is oxidizer mass flow, represent the command parameter of the system. 
Altogether by simple geometrical reasoning, volume element is:

$\mathrm{d} V=2 \pi r L \mathrm{~d} r$,

and the variation in time is given by:

$\dot{V}=2 \pi r L \dot{r}$,

where $L$ is length of the fuel grain.

Substituting (3) in (5) we obtain:

$\dot{V}=L a 2^{l+1} \pi^{-n+1} \dot{m}_{o x}^{n} p^{m} r^{l-2 n+1}$.

Moreover, taking into account that:

$r=V^{1 / 2}(2 \pi L)^{-1 / 2}$,

we obtain the volume equation:

$\dot{V}=c_{v o l} \dot{m}_{o x}^{n} V^{-n+1 / 2+l / 2} p^{m}$,

where the volume coefficient is:

$c_{v o l}=a L^{n+1 / 2-l / 2} 2^{n+1 / 2+l / 2} \pi^{1 / 2-l / 2}$.

This assumes an ideal burning, meaning that the burn advances equally throughout the length of the fuel grain with no erosion along the burning port. In reality, the burning port does not maintain the initial geometry and instead of it starts to diverge near the injection valve. Expression (6) can also be generalized for non-circular combustion ports where $r$ would be substituted by general shape functions describing the shape of each individual combustion port.

Using the continuity equation, the variation of the mass in the burning chamber is the difference between the mass produced in time unit by burning the fuel and the mass that exits the motor through the nozzle in time unit:

$\frac{\partial(\rho V)}{\partial t}=\dot{m}_{\text {in }}-\dot{m}_{\text {out }}$,

where $V$ is the volume of the burning chamber, and $\rho$ is gas density inside the burning chamber, $\dot{m}_{i n}$ is the input mass generated from the combustion of fuel inside the motor chamber and $\dot{m}_{\text {out }}$ is the output mass ejected through the nozzle of the rocket motor. The input mass per time unit is given by the oxidizer flow rate and fuel 
consumption rate (strictly correlated with the regression rate of the given fuel/oxidizer pair):

$\dot{m}_{i n}=\dot{m}_{o x}+\dot{m}_{f}$,

and the output mass in time unit is the exit through the nozzle:

$\dot{m}_{\text {out }}=\Lambda A_{t} \sqrt{p \rho}$,

where, $A_{t}$ is the throat area, $p$ is chamber pressure and

$$
\Lambda=\sqrt{k(2 /(k+1))^{(k+1) /(k-1)}} .
$$

Taking into account that the fuel consuming mass in time unit is:

$$
\dot{m}_{s f}=\rho_{f} \dot{V}
$$

Developing relation (10) we obtain density equation:

$$
\dot{\rho}=\frac{\dot{m}_{o x}}{V}+\left(\rho_{f}-\rho\right) \frac{\dot{V}}{V}-\frac{\Lambda A_{t}}{V} \sqrt{p \rho} \text {. }
$$

On the other hand, from equation (8) we have:

$$
\frac{\dot{V}}{V}=c_{v o l} \dot{m}_{o x}^{n} p^{m} V^{-n-1+(1+l) / 2}
$$

and the density equation became:

$$
\begin{aligned}
\dot{\rho} & =\dot{m}_{o x} V^{-1}+\left(\rho_{f}-\rho\right) c_{v o l} \dot{m}_{o x}^{n} V^{-n-1 / 2+l / 2} p^{m}- \\
& -\Lambda A_{t} V^{-1} p^{1 / 2} \rho^{1 / 2}
\end{aligned}
$$

Beside the volume equation (8) and density equation (17), the third equation expressing the change in temperature or pressure of the combustion products we need.

We consider the input energy for the system is the heat quantity $Q_{C}$ educts by burning reaction of $m_{f}$ solid fuel.

Also, we take into account that the specific heat at constant volume $C_{V}$ can be obtain from the relation: 
$C_{V}=R /(k-1)$.

To build the temperature equation, we start from the following relationship of energy balance:

$\mathrm{d} U=\mathrm{d} U_{1}+\mathrm{d} U_{2}+\mathrm{d} U_{3}+\mathrm{d} U_{4}$,

where the reaction energy of the fuel is given by:

$$
\mathrm{d} U=Q_{C} \mathrm{~d} m_{f},
$$

is converted into:

- internal energy growth due to additional gas from the combustion chamber:

$$
\mathrm{d} U_{1}=C_{V} T V \mathrm{~d} \rho=R(k-1)^{-1} T V \mathrm{~d} \rho ;
$$

- energy in gas from the combustion chamber increased due to temperature variation:

$\mathrm{d} U_{2}=\rho V C_{V} \mathrm{~d} T=R(k-1)^{-1} \rho V \mathrm{~d} T ;$

- kinetic energy due to gas flow:

$\mathrm{d} U_{3}=k(k-1)^{-1} R T \mathrm{~d} m_{\text {out }}$.

- loss of energy due to the disposal of heat through the chamber walls:

$\mathrm{d} U_{4}=q \mathrm{~d} t$,

where $q$ is the amount of heat transferred to the combustion chamber in time unit (heat flow) $[\mathrm{J} / \mathrm{s}]$. If we take the derivative of (19) with respect to time and then simplify it, we obtain:

$Q_{C} \dot{m}_{f} / C_{V}=T V \dot{\rho}+\rho V \dot{T}+k T \dot{m}_{\text {out }}+q / C_{V}$,

hence we obtain the temperature equation:

$$
(k-1) Q_{C} \frac{\rho_{f}}{p} \frac{\dot{V}}{V}=\frac{\dot{\rho}}{\rho}+\frac{\dot{T}}{T}+\frac{k \Lambda A_{t}}{V} \sqrt{\frac{p}{\rho}}+\frac{(k-1) q}{p V} .
$$


Taking into account that the state equation can be written in form:

$\dot{p} / p=\dot{\rho} / \rho+\dot{T} / T$,

we transform the temperature equation (26) into the pressure equation:

$$
\begin{gathered}
\dot{p}=(k-1) \rho_{f} Q_{C} c_{v o l} \dot{m}_{o x}^{n} V^{-n-1 / 2+l / 2} p^{m}- \\
-k \Lambda A_{t} V^{-1} \rho^{-1 / 2} p^{3 / 2}-(k-1) q V^{-1}
\end{gathered} .
$$

Having differential equations (17) and (28) solved, for temperature we can use state relation:

$T=p /(R \rho)$.

Using paper [6] we obtain the rate between throat area $A_{t}$ and exit area of the nozzle $A_{e}$ propose the relation:

$$
\frac{A_{t}}{A_{e}}=\sqrt{\frac{2}{k-1}\left(\frac{2}{k+1}\right)^{\frac{k+1}{1-k}} \tilde{p}_{e}^{\frac{2}{k}}\left(1-\tilde{p}_{e}^{\frac{k-1}{k}}\right)},
$$

with the relative pressure is given by:

$\tilde{p}_{e}=p_{e} / p$

where $p_{e}$ is gas pressure in exit area.

Assuming constant ratio of specific heats throughout the expansion process, one finds the thrust force relation indicated in paper [4-5]:

$F=A_{e} p_{H}\left[\frac{p}{p_{H}} \sigma_{c} \frac{A_{t}}{A_{e}}\left(\frac{2}{k+1}\right)^{\frac{1}{k-1}} \lambda-1\right]$,

where $p_{H}$ is atmospheric pressure, and $\sigma_{c}$ is overall loss of thrust by nozzle and $\lambda$ is the ratio of the combustion gas velocity in the nozzle exit plane and, respectively, nozzle throat plane.

The oxidizer feeding is assumed to take place at constant pressure as opposed to simpler hobby HRM that have the oxidizer self-pressurized. In our case the LOX is injected using turbo-pumps electronically controlled. By controlling the mass flow of 
oxidizer one can control the thrust of the engine since the oxidizer mass flow is a control parameter [5].

\section{Thermochemistry-Solid Methane and LOX}

In our model, we assume solid methane to have the same caloric power but with a different density (higher density) than liquid methane.

According to NIST chemistry database, the methane has a triple point at around $p=0.1169 \mathrm{~atm}$ and $T=90 \mathrm{~K}$. Since during the operation of the motor the pressure and temperature conditions are not, under the above triple point values, then we assume that sublimation, does not take place and, hence, the methane suffers a three-phase transformation: solid -> liquid -> gas.

Thus during the combustion there will be a liquid methane layer at the surface of the solid methane grain. This liquid layer vaporizes and interacts with the oxidizer burning inside the combustion chamber.

Transformations from solid to liquid and from liquid to gas require an amount of energy equal to the sum of fusion and vaporization enthalpies.

In our algorithm, we used the main thermo-chemical relation:

$$
\sum_{R} n_{i}\left(h_{f}+\Delta h\right)_{i}=\sum_{P} n_{e}\left(h_{f}+\Delta h\right)_{e}
$$

where $\Delta h$ represents the change in enthalpy from a reference temperature, typically 298 K. In our computations, we assumed that initially the reactants are at an initial temperature equal with the reference temperature and hence the left hand-side $\Delta h$ terms are all equal with zero. Using the conservation of mass in equation (33) and the values for enthalpies that can be found in NIST Chemistry tables one can compute the adiabatic flame temperature for certain reactants and products of reaction. The adiabatic flame temperature assumes no heat is loss to the environment and that the chemical composition of products is maintained constant throughout the reaction. These conditions are considered to be chemical and thermal equilibrium and under these conditions the adiabatic flame temperature represents the highest temperature that can be obtained with certain reactants. More than this, the adiabatic flame temperature is reached for stoichiometric ratios of the reactants mixture.

Thermo-chemically we have the following relations for the temperature and pressure in the critical section of the nozzle:

$$
T^{*}=\frac{T_{0}}{1+\frac{k-1}{2}}
$$




$$
p^{*}=\frac{p_{0}}{\left[1+\frac{k-1}{2}\right]^{\frac{k}{k-1}}}
$$

Also, the specific impulse and the characteristic velocity have the following thermochemical formulations:

$$
\begin{gathered}
\left.I_{s p}=\frac{1}{g} \sqrt{2 T_{0} \frac{R^{\prime}}{M}\left(\frac{k}{k-1}\right)\left[1-\left(\frac{p_{e}}{p_{0}}\right)^{\frac{k-1}{k}}\right.}\right] \\
c^{*}=\sqrt{\frac{\left(R^{\prime} / M\right) T_{0}}{k\left(\frac{2}{k+1}\right)^{\frac{k+1}{k-1}}}}
\end{gathered}
$$

From previous works [5-7] we can determine the optimum O/F ratio in order to maximize the Isp of the HRM. We observe that the peak performance is obtained near the ratio O/F equal with 3 .

In Fig. 1, we show the dependence of specific impulse on pressure for a fixed mass ratio between methane and liquid oxygen. In this case the $\mathrm{O} / \mathrm{F}$ ratio is considered to be 3 which is closed to the stoichiometric ratio $\sim 4$.

In Fig. 2 we represent the temperature variation with $\mathrm{O} / \mathrm{F}$ ratio and we observe that the temperature increases as we approach stoichiometric $\mathrm{O} / \mathrm{F}$ ratio.

While specific impulse has a maximum value, other constructive considerations should be taken into account, e.g. increase of temperature with the increase of specific impulse.

In TABLE 2 we show a comparison performance chart that basically shows specific impulse obtained at $100 \mathrm{~atm}$ for various oxidizers used in combination with methane at ideal (stoichiometric) chemical ratios. We have assumed exit pressure $1 \mathrm{~atm}$ and area ratio to be 10 .

We can observe that the LOX/solid methane is the highest Isp achiever and is a good candidate for the upper stages of a launch vehicle.

\section{DOF MODEL}

The flight dynamics of the launcher can only be modeled by a full 6 DOF model that takes into account both the Earth rotations and the variation of gravitational acceleration with altitude and latitude.

In order to study the motion of the vehicle we employ two frames of reference: the body frame and the Earth frame. 
The body frame is fixed with respect to the vehicle having the $X$ axis pointing along the longitudinal axis of the vehicle while the $Y$ axis points along the starboard of the vehicle. The Z-axis is chosen conventionally to point straight down.

The Earth reference frame is attached to the Earth (usually its origin coincides with the launch point) having the $X$ positive axis pointing towards the North and the $Y$ positive axis pointing towards East. The $Z$ axis points upward indicating the local vertical. The relation between Earth (ground) reference frame and body reference frame is shown in Fig. 3

The orientation of the body reference frame with respect to the Earth reference frame is described by the angle triplet: $\phi, \Theta, \psi$ and the transition between the Earth and the body reference frame can be done using a rotation matrix. We have used 6 DOF equations as shown in [8]

Considering the body reference frame one can describe the motion of the vehicle by using the following 6 equations (one for each degree of freedom). Three equations are written for the translations:

$$
\begin{aligned}
& \dot{u}=\frac{1}{m}\left(T+F_{\text {axial }}\right)-g \sin \theta+r v-q w \\
& \dot{v}=\frac{1}{m} F_{\text {side }}+g \cos \theta \sin \varphi+p w-r u \\
& \dot{w}=\frac{1}{m} F_{\text {normal }}-g \cos \theta \cos \varphi+q u-p v
\end{aligned}
$$

Another three equations are written for the rotation dynamics:

$$
\begin{aligned}
& \dot{p}=\frac{1}{I_{x x}}\left[M_{\text {propulsion }}+M_{x}+\left(I_{y y}-I_{z z}\right)\right] \\
& \dot{q}=\frac{1}{I_{x x}}\left[M_{y}+\left(I_{z z}-I_{x x}\right)\right] \\
& \dot{r}=\frac{1}{I_{x x}}\left[M_{z}+\left(I_{x x}-I_{y y}\right)\right]
\end{aligned}
$$

The most difficult part in numerical estimations is to obtain valid aerodynamic coefficients for the chosen vehicle. One method to obtain these coefficients is to use the dedicated software MISSILE DATCOM that can offer tabulated coefficients for a wide range of flight envelopes.

Another option is to use a semi-analytical approach and consider semi-empirical relations for each of the coefficients. The advantage of this method is the readily availability and the possibility to obtain fast results. However, the precision of the semi-analytical approach might be lower than the MISSILE DATCOM approach. 


\section{NUMERICAL RESULTS}

Two main strategies from the numerical point of view can be used. One of them involves computing the accelerations in the body frame using equations (37) through (42). Then the accelerations can be integrated once using Runge-Kutta 4 obtaining the velocities in the body frame. The angular velocities are transformed at this point to the rotating Earth reference frame by using relation (49). The velocity field can be rotated using the rotation matrix $T$ and then integrated again to find the positions and the attitude angles.

Another numerical strategy involves finding the accelerations by using the equations (37) through (42) without using the cross term products in the translation acceleration relations.

Hence, the new translation accelerations in the body frame are given by the relations:

$$
\begin{aligned}
& \dot{u}=\frac{1}{m}\left(T+F_{\text {axial }}\right)-g \sin \theta \\
& \dot{v}=\frac{1}{m} F_{\text {side }}+g \cos \theta \sin \varphi \\
& \dot{w}=\frac{1}{m} F_{\text {normal }}-g \cos \theta \cos \varphi
\end{aligned}
$$

The angular accelerations are not modified and, hence, are still given by the equations (40) through (42).

Next, the body frame accelerations are rotated through the rotation matrix (19) to the Earth reference frame and then integrated twice by using Runge-Kutta 4. An important thing to note for this method is that the angular velocities after being transformed to the rotating Earth reference frame has to be rotated back from Earth reference frame to body reference frame and then integrated once in order to find the attitude angles. Both methods are mathematically equivalent and should provide similar results.

We performed first few numerical runs for simulated suborbital flights aiming to maximize the microgravity time available for the payload.

Hence, we have in Fig. 4 the velocity of the vehicle as a function of time. The time is represented in fractions of the time to orbit (1.0 on time axis represents 8 minutes) and the velocity in fractions of the needed orbital velocity (1.0 on velocity axis represents $7.9 \mathrm{~km} / \mathrm{s}$ ).

In Fig. 5 we show the variation of mass of the launcher with the obvious decrease of mass during each stage operation. The mass is represented in fractions of the total mass while the time is represented in fractions of the total time to orbit (1.0 on time axis represents 8 minutes). The coasting phase between each stage separation is long enough to allow re-alignment of certain sensors and safe cold separation. 


\section{GUIDANCE SYSTEMS}

For the guidance of the launcher, we propose an inertial navigation platform capable to produce a full attitude solution and generating guidance controls for the jet vanes and the guidance micro-thrusters.

Nowadays many COTS inertial navigation platforms are available and many of them fit the requirements for a low cost orbital launcher.

As inertial autopilots we have in Figs. 6, 7, 8 several commercial available units. These are used for both UAV and cruising missile while they contain only commercial components [9-11].

The typical autopilot uses Kalman filtering and quaternion formulation in order to obtain the full attitude solution for the launcher. The processor fuses the inertial data with an external reference.

All the above units are tactical grade IMU. They can be used as autopilots only by having an external reference. The most common external reference is a GPS receiver that updates the inertial solution correcting the drift that occurs over time.

However, other types of external references can be used. For example, magnetic compass and radio altimeter can provide both the heading and the altitude reference compensating the lack of a GPS receiver or of usable GPS signal.

\section{CONCLUSIONS}

In this paper, we described a new launcher concept using a solid-hybrid-hybrid three stage structure. The solid rocket motor is an excellent candidate for the first stage of the vehicle while the hybrid propulsion assures simplicity and low cost characteristics for the upper stage. The hybrid rocket motor is proposed to use LOX and solid methane for high performance operation. An internal ballistic model for a cryogenic hybrid rocket motor is presented together with a full 6 DOF model for the flight dynamics of the vehicle.

We also presented several commercial autopilots that can be used as inertial navigation units for all the three stages of the launcher.

Future work on guidance system using micro-thrusters is intended as part of separate paper and funded through a separate project.

\section{ACKNOWLEDGMENTS}

The author thanks to Romanian Space Agency (ROSA) for the continuous support for this research work part of the finance support being offered through the Vector for Satellite Launcher- national research project funded through the grant: 82-67/2008. 


\section{REFERENCES}

[1] Chelaru T.V. Mingireanu F., "Hybrid rocket engine, theoretical model and experiment", 60th International Astronautical Congress, ISSN 1995-6258, Daejeon, Republic of Korea, 12-16 October 2009

[2] Sutton, G. P. , Biblarz, O.,"Rocket propulsion elements", 7nt ed.,John Wiley\&Sons Inc.ISBN 0-471-32642-9, New York, 2001.

[3] Collburn,B., "A manual for hybrid propulsion system design", $1^{\text {st }}$ ed, Aerocon Systems, 2006.

[4] Christopher P. St.Clair; Eric E. Rice; William H. Knuth; Daniel J. Gramer, "Advanced Cryogenic Solid Hybrid Rocket Engine Developments: Concept and Test Results", 34 ${ }^{\text {th }}$ AIAA/ASME/SAE/ASEE Joint propulsion conference \& exhibit, July 13-15, 1998, Cleveland, OH, USA.

[5] Laforce,P. D., "Technological development of a throttling hybrid propulsion system", UTC 2215-FR, January 1967.

[6] .A. Klepikov, B.I. Katorgin, V.K. Chvanov, "The new generation of rocket engines, operating by ecologically safe propellant liquid oxygen and liquefied natural gas(methane)", Acta Astronautica, 41 p. 209-217, 1997.

[7] Mingireanu Florin, Marius Stoia-Djeska, "Methane based cryogenic hybrid rocket motor. Oxidizer doping", International Astronautical Congress 2011, Cape Town, South Africa.

[8] Qian Xinfang. "Flying dynamics of missile", Beijing, Publishing House of Beijing institute of technology, 2000.

[9] https://aerospace.honeywell.com/en/products/navigation-andsensors/hg1700

[10] http://www.moog-crossbow.com/products/inertial-products/

[11] http://www.sbg-systems.com/

Table 1. Proposed HRM launcher.

\begin{tabular}{|l|c|c|c|}
\hline Propulsion & Solid & Hybrid & Hybrid \\
\hline Thrust (kN) & 45.5 & 25.5 & 5 \\
\hline Burn time (s) & 100 & 180 & 240 \\
\hline Isp (s) & 270 & 310 & 310 \\
\hline
\end{tabular}

Table 2. Comparison performance.

\begin{tabular}{|l|c|}
\hline O/F pair & Isp (s) \\
\hline LOX/methane & $295-310$ \\
\hline Nitrate acid/methane & 215 \\
\hline Oxychlorine trifluoride/methane & 206 \\
\hline Oxygen difluoride/methane & 254 \\
\hline
\end{tabular}




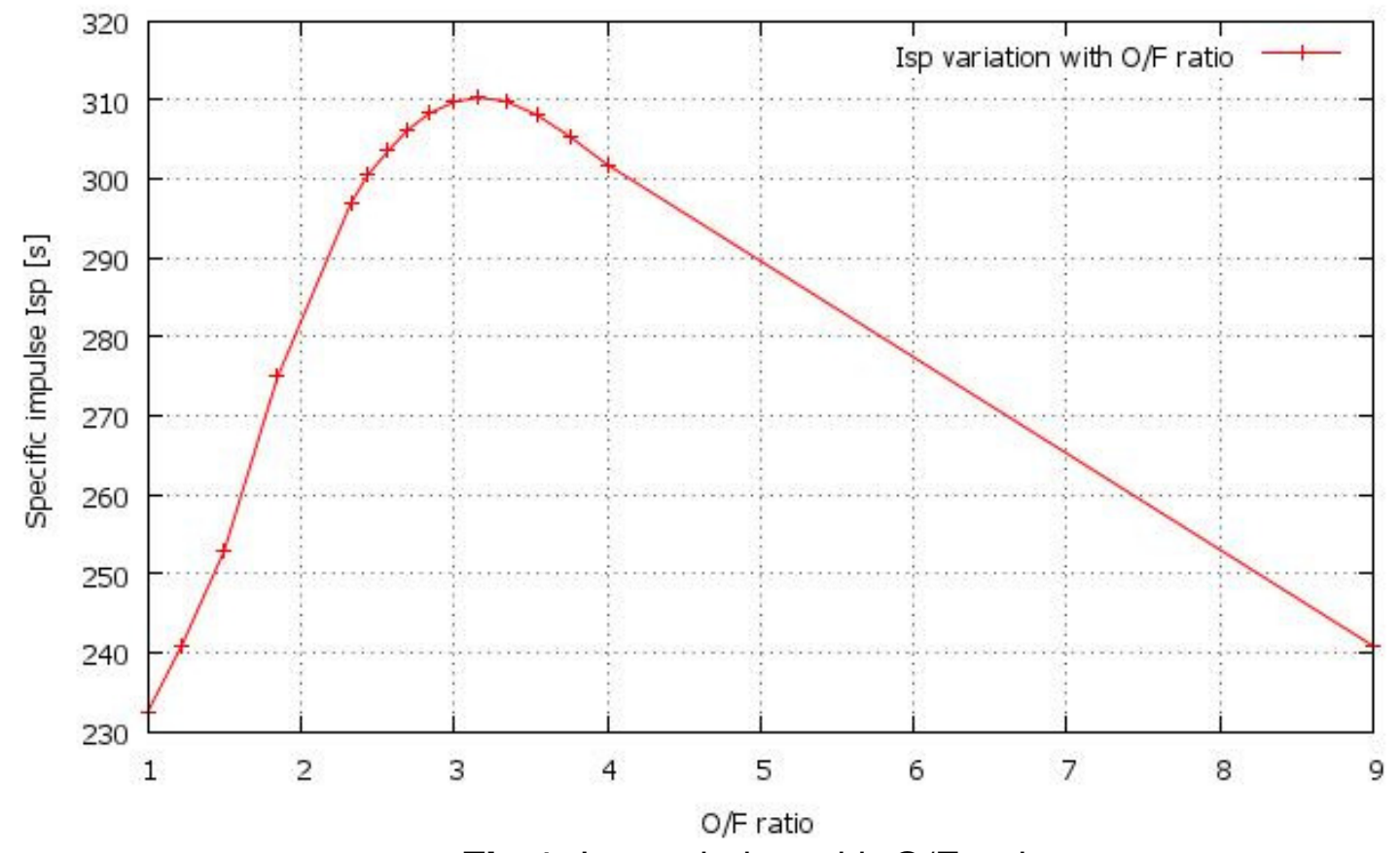

Fig.1. Isp variation with $\mathrm{O} / \mathrm{F}$ ratio.

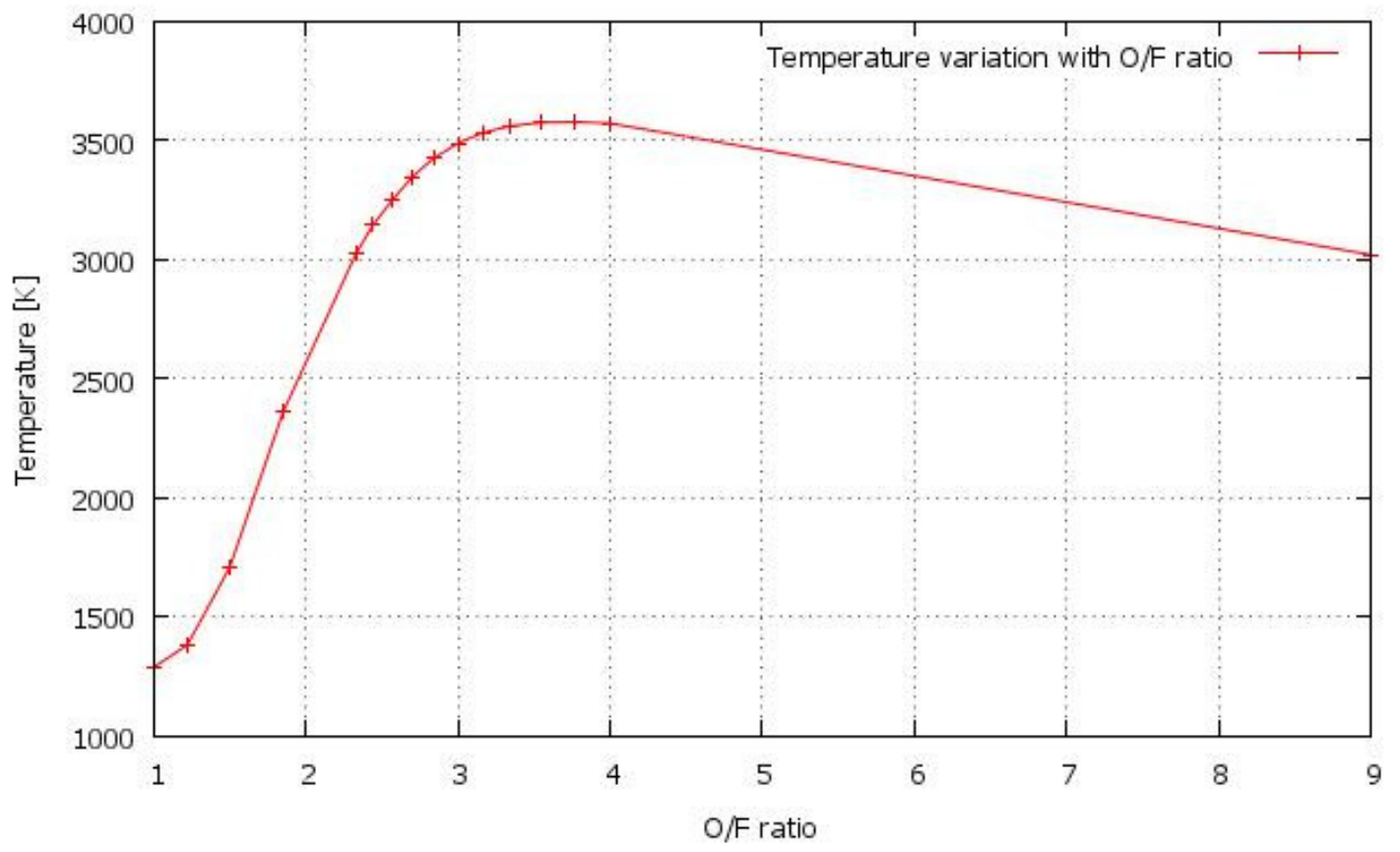

Fig.2. Temperature variation with $\mathrm{O} / \mathrm{F}$ ratio. 


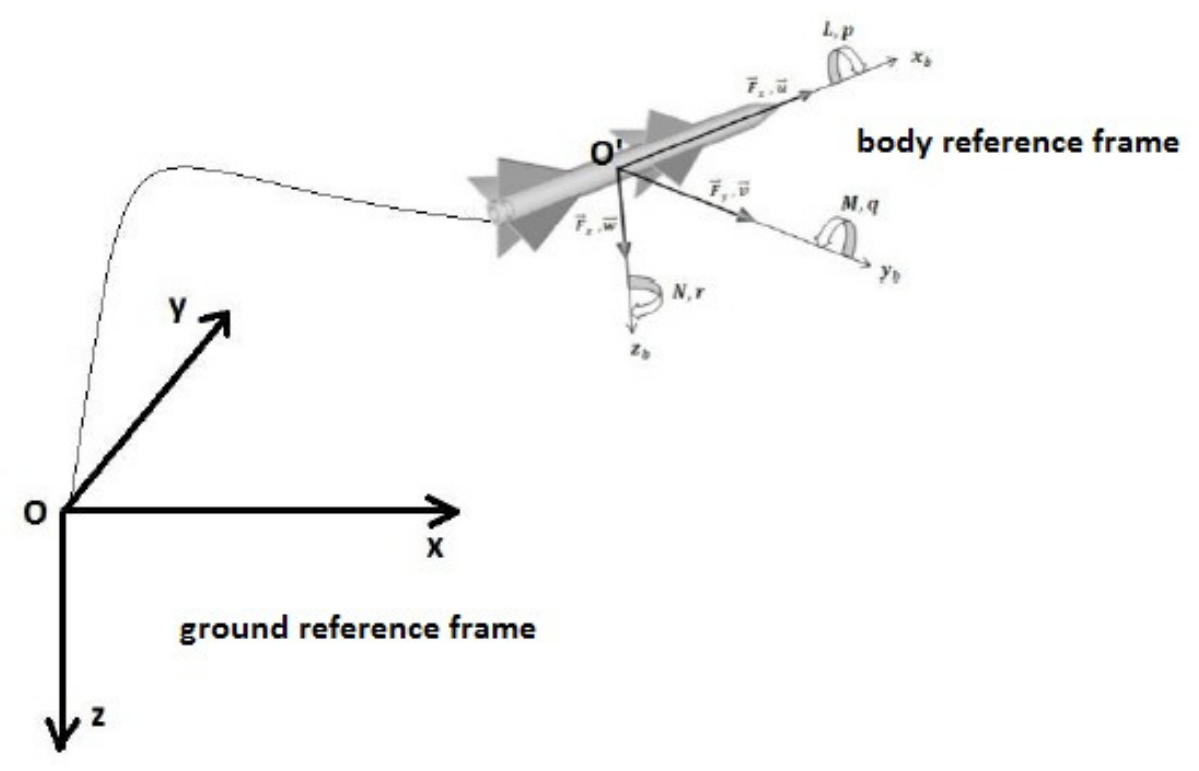

Fig. 3. Reference frames.

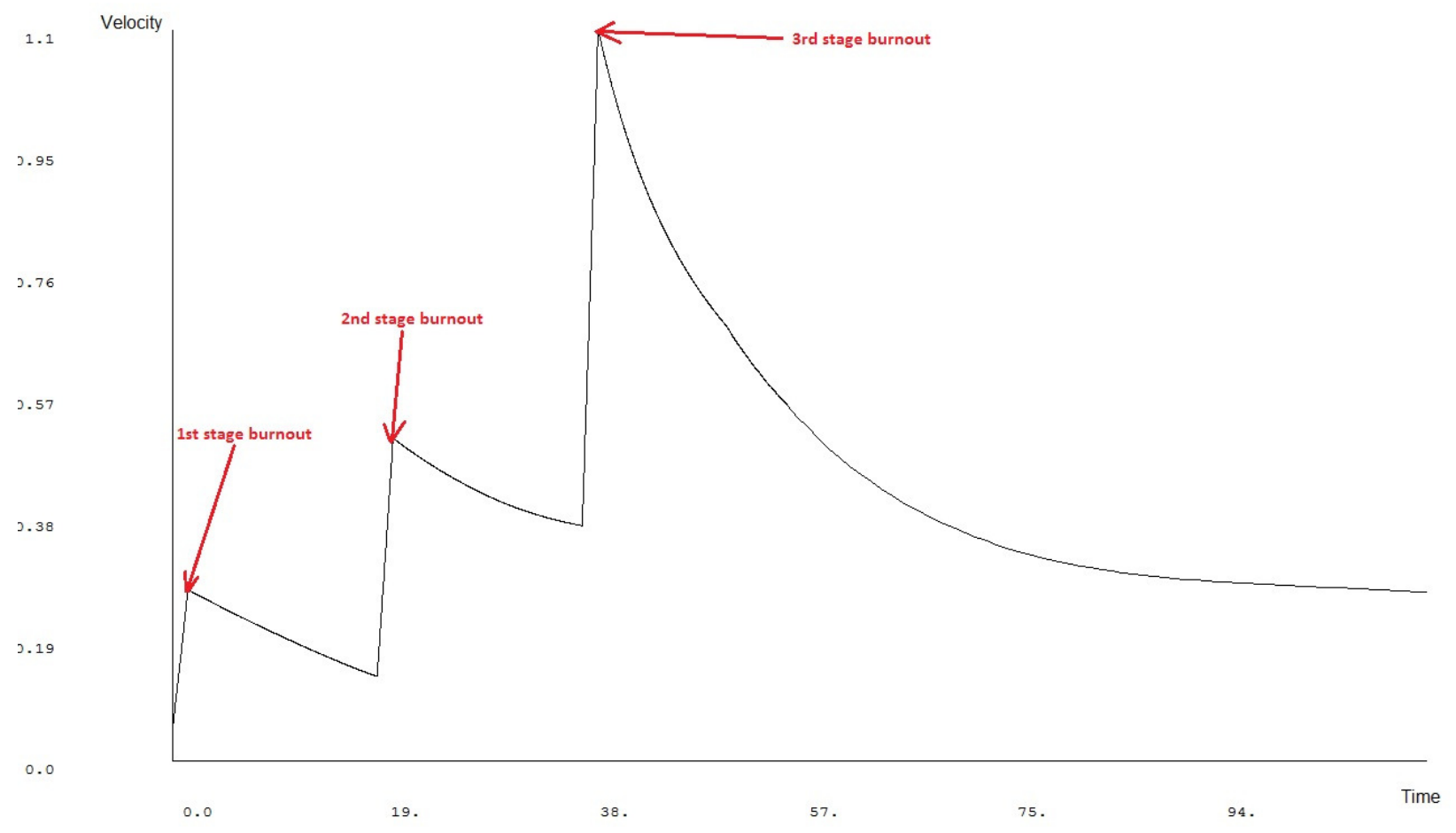

Fig. 4. Velocity as a function of time. 


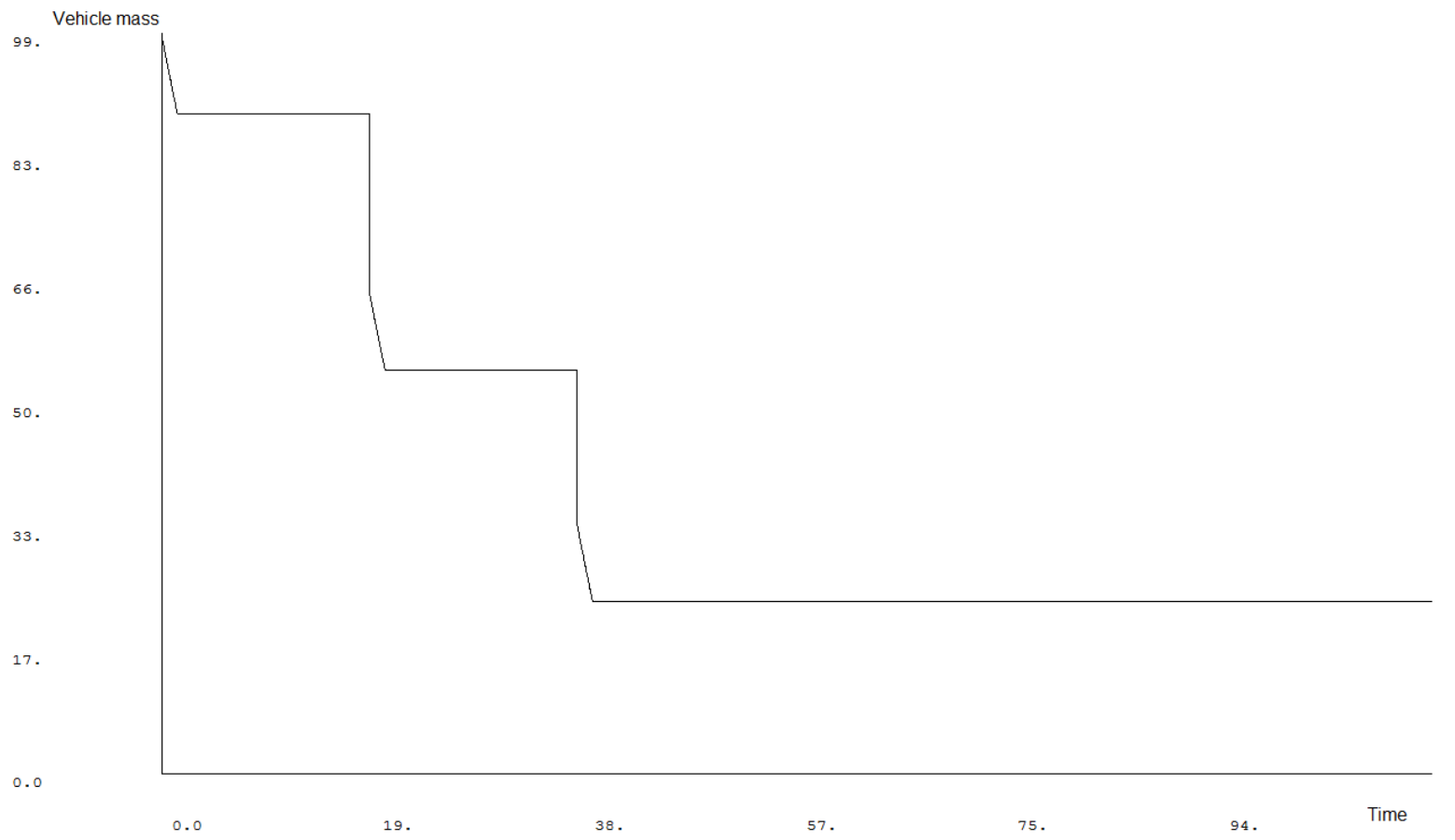

Fig. 5. Mass variation during stage operation.

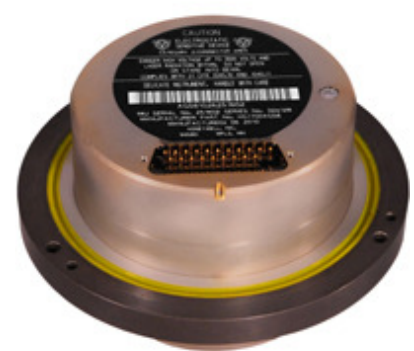

Fig. 6. HG 1700 Honeywell Inertial Measurement Unit.

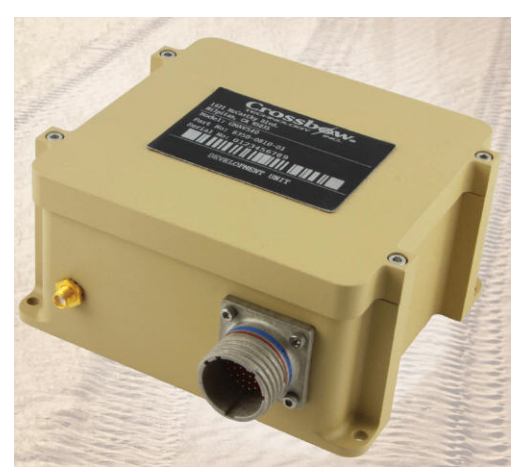

Fig. 7. XBOW Inertial Navigation Unit. 


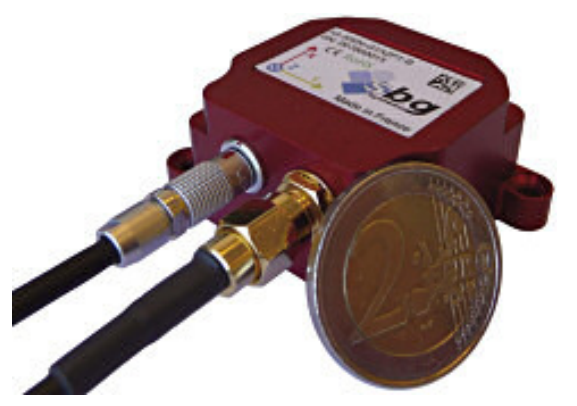

Fig. 8. SBG Inertial Navigation Unit. 
